\title{
A GENERALIZATION OF ABSOLUTE RETRACTS
}

\author{
JOHN R. MARTIN 1
}

\begin{abstract}
In this paper the concept of an absolute retract is generalized to a new concept which we call an absolute approximate retract. It is shown that for the class of compact metric spaces, the class of absolute approximate retracts contains the class of absolute retracts and lies in the class of contractible Peano continua.
\end{abstract}

1. Notation. All topological spaces considered in this paper will be Hausdorff spaces. The symbol $Q$ shall be used to denote one of the following classes of spaces: compact metric, separable metric, metric, compact, regular Lindelöf, fully normal. Definitions for these spaces may be found in [2] and we shall adopt the notation used in [2]. In particular, we shall let $\operatorname{AR}(Q)$ (respectively $\operatorname{ANR}(Q)$ ) denote the class of absolute (neighborhood) retracts relative to $Q$.

The closure of a subset $A$ of a topological space shall be denoted by $\mathrm{Cl} A$.

\section{Approximate retracts.}

Definition. A subset $A$ of a space $X$ is called an approximate retract of $X$ if for every neighborhood $U$ of $A$ in $X$ there is a retract $R$ of $X$ such that $A \subset R \subset U$.

The following two lemmas show that an approximate retract of a continuum is itself a continuum.

Lemma 1. An approximate retract of a Hausdorff space $X$ is closed in $X$.

Proof. Let $A$ be an approximate retract of a Hausdorff space $X$. Suppose $A$ is not closed in $X$. Let $p \in \mathrm{Cl} A-A$. Then there is a retract $R$ of $X$ such that $A \subset R \subset X-\{p\}$. Since $R$ must be closed in $X[1, \mathrm{p} .10]$, it follows that $p \in R$. This contradiction shows that $A$ is closed in $X$.

Lemma 2. An approximate retract of a connected normal space is connected.

Proof. Let $A$ be an approximate retract of a connected normal space $X$.

Received by the editors July 16, 1974.

AMS (MOS) subject classifications (1970). Primary 54C15, 54C55.

Key words and phrases. Retract, absolute retract, absolute neighborhood retract, Peano continuum.

1 The research for this article was supported in part by the National Research Council of Canada (Grant A8205). 
Suppose $A$ is not connected. By Lemma $1, A$ is closed in $X$. Hence there exists two disjoint nonempty closed subsets $A_{1}, A_{2}$ in $X$ such that $A=$ $A_{1} \cup A_{2}$. Since $X$ is normal, there exist disjoint open sets $U_{1}$ and $U_{2}$ in $X$ such that $A_{1} \subset U_{1}$ and $A_{2} \subset U_{2}$. Let $U=U_{1} \cup U_{2}$. Then there is a retract $R$ of $X$ such that $A \subset R \subset U$. But $R=\left(R \cap U_{1}\right) \cup\left(R \cap U_{2}\right)$, which contradicts the fact that a retract of a connected space is connected. Therefore $A$ must be connected.

Examples. (1) Let $X$ denote the closure of the curve in the plane $E^{2}$ whose equation is given by $y=\sin x^{-1}, 0<x \leq 1 / \pi$. It is easy to see that every neighborhood of $X$ in $E^{2}$ contains an AR (compact metric)-space which contains $X$, and hence $X$ is an approximate retract of $E^{2}$. Thus, unlike the case for retracts of $E^{2}$, a compact approximate retract of the plane need not be locally connected or even arcwise connected.

(2) S. Kinoshita [4] and J. M. Lysko [5] have given examples of contractible continua in 3-space $E^{3}$ which do not have the fixed point property. It is easy to show that their examples are approximate retracts of $E^{3}$. Consequently, a compact contractible approximate retract of $E^{3}$ need not have the fixed point property (even with respect to homeomorphisms).

\section{Absolute approximate retracts.}

Definition. A space $X$ is an absolute approximate retract relative to the class $Q$ (abbreviated $\operatorname{AAR}(Q)$ ) if $X \in Q$ and whenever $X$ is embedded as a closed subset of a $Q$-space $Z$, then $X$ is an approximate retract of $Z$.

\section{Proposition 1. Every AAR(Q)-space is contractible.}

Proof. First we consider the case where $Q$ is not the class of separable metric or metric spaces. Let $X$ be an $\operatorname{AAR}(Q)$-space. Then $X \in Q$ and, if $I$ denotes the closed unit interval $[0,1]$, it is well known that $X \times I \in Q$ [2, p. 331]. Let $C(X)$ denote the cone over $X$ with vertex $p$. Since $C(X)$ is the Hausdorff image of a closed map from the $Q$-space $X \times I$, it follows that $C(X) \in Q$ [7]. Moreover, $X$ is homeomorphic to the closed subset $X \times\{0\}$ of $C(X)$. Thus there is a retract $R$ of $C(X)$ such that $X \times\{0\} \subset R \subset C(X)-\{p\}$.

Define a retraction $r: R \rightarrow X \times\{0\}$ by $r(x, t)=(x, 0)$ for all $(x, t)$ in $R$. Since $X \times\{0\}$ is a retract of $R$ and $R$ is a retract of $C(X)$, it follows that $X \times\{0\}$ is a retract of $C(X)$. The point $p$ is a strong deformation retract of $C(X)[3, \mathrm{p} .19]$, and thus $C(X)$ is contractible. It then follows that $X \times\{0\}$ is contractible $[1$, p. 26], and therefore $X$ is contractible.

Now we consider the case where $Q$ denotes the class of separable metric or metric spaces. Suppose $X$ is an AAR(Q)-space. Then, by the Kuratowski-Wojdysławski embedding theorem [1, p. 79], we may assume that $X$ is embedded as a bounded subset of a proper linear subspace $L$ of a Banach space $B$. Let $p \in B-L$, and let $C(X)$ denote the subset of $B$ consisting of the 
union of all straight line intervals $[p, x]$ where $x \in X$. Then $C(X)$ is a contractible $Q$-space, and the remaining argument is analogous to the argument used for the other cases.

As a corollary to Proposition 1, we obtain the following result.

Theorem 1. A space $X$ is an $\operatorname{AR}(Q)$-space iff $X$ is an $\operatorname{AAR}(Q)$-space and $X$ is an $\operatorname{ANR}(Q)$-space.

Proof. It is clear that if $X$ is an $A R(Q)$-space, then $X$ is an $\operatorname{AAR}(Q)$ space and $X$ is an $\operatorname{ANR}(Q)$-space.

Now suppose $X \in \operatorname{AAR}(Q)$ and $X \in \operatorname{ANR}(Q)$. Then, by Proposition 1, $X$ is a contractible $\operatorname{ANR}(Q)$-space. Therefore $X$ is an $\operatorname{AR}(Q)$-space [2, p. 332].

Proposition 2. Every first countable AAR(Q)-space is locally connected.

Proof. Suppose $X$ is a first countable $\operatorname{AAR}(Q)$-space which is not locally connected at a point $p$. Then there exists a neighborhood $V$ of $p$ in $X$ and points $p_{1}, p_{2}, \ldots, p_{i}, \ldots$ such that $\lim _{i \rightarrow \infty} p_{i}=p$, and each of the points $p, p_{1}, p_{2}, \ldots, p_{i}, \ldots$ lies in a different component of $V$. To complete the proof of Proposition 2, we construct a $Q$-space $Z$ such that $X$ is a closed subset of $Z$ which is not an approximate retract of $Z$. The space $Z$ is an identification space formed from $X$ and a plane continuum $Y$.

We now construct the plane continuum $Y$. Let $q_{i}$ denote the point $(1 / i$, $0), i=1,2, \ldots$, and let 0 denote the origin in the plane $E^{2}$. For each $i=$ $1,2, \ldots$, let $A_{i}$ denote the curve in $E^{2}$ whose equation is given by

$$
y=\frac{1}{i} \sin \left(\frac{\pi}{i(i+1) x-i}\right), \quad \frac{1}{i+1}<x \leq \frac{1}{i} .
$$

Then $\mathrm{Cl} A_{i}$ is a plane continuum joining the point $q_{i}$ to a vertical interval of length $2 / i$ which passes through $q_{i+1}$. Define $B=\bigcup_{i=1}^{\infty} \operatorname{Cl} A_{i} \cup\{0\}$. Let $A$ be a set lying in $E^{2}$ homeomorphic to a half-open interval such that $A$ is disjoint from $B$ and "converges" to $B$ in such a way that the following properties are satisfied.

(1) There is a sequence of arcs $S_{1}, S_{2}, \ldots$, filling up $A$ such that the interiors of any two consecutive arcs lie on opposite sides of the $x$-axis.

(2) $\lim _{j \rightarrow \infty} S_{2 j-1}=B$ and $\lim _{j \rightarrow \infty} S_{2 j}=B$.

Define $Y=A \cup B$.

Now lat $C=\bigcup_{i=1}^{\infty}\left\{q_{i}\right\} \cup\{0\}$, and let $f: C \rightarrow X$ be the map defined by $f(0)=p, f\left(q_{i}\right)=p_{i}$ for $i=1,2, \ldots$. The space $Z$ is obtained by taking the disjoint union of $Y$ and $X$, and then identifying each point $y \in C$ with its image $f(y) \in X$. Then $Z$ is a $Q$-space and $X$ is a closed subset of $Z$.

We first note that only finitely many of the sets $\mathrm{Cl} A_{i}$ can be retracted into $X$. To see this, suppose $r$ is a retraction mapping infinitely many of the sets $\mathrm{Cl} A_{i}$ into $X$. Now $r$ is continuous at $p$, so there exists a neigh- 
borhood $U$ of $p$ in $Z$ such that $r(U) \subset V$. Since $U$ must contain all but finitely many of the sets $\mathrm{Cl} A_{i}$, it follows that for some $j, r\left(\mathrm{Cl} A_{j}\right) \subset V$. But then $r\left(\mathrm{Cl} A_{j}\right)$ is a connected subset of $V$ containing $p_{j}$ and $p_{j+1}$, which is a contradiction.

Now let $W$ be a neighborhood of $X$ in $Z$ such that any point in $W \cap Y$ has distance less than $1 / 2$ from the $x$-axis. Then $W$ contains infinitely many of the sets $\mathrm{Cl} A_{i}$, and no connected subset of $W$ containing $X$ contains a point of $A$. Suppose $R$ is a retract of $Z$ such that $X \subset R \subset W$. Since $Z$ is connected and only finitely many of the sets $\mathrm{Cl} A_{i}$ can be retracted into $X$, it follows that $R$ must be a connected subset of $W$ containing infinitely many of the sets $\mathrm{Cl} A_{i}$. But if $\mathrm{Cl} A_{j} \subset R$, then a subinterval of $A$ must be mapped onto the nonlocally connected space $\mathrm{Cl} A_{j}$, which is impossible. Therefore $X$ is not an approximate retract of $Z$ and the proof is complete.

In the following discussion we shall restrict ourselves to the compact metric case and omit the symbol $Q$. Using this notation, we obtain the following theorem from Propositions 1 and 2.

Theorem 2. Every AAR-space is a contractible Peano continuum.

Corollary 1. The 1-dimensional AAR-spaces coincide with the 1-dimensional. AR-spaces.

Corollary 2. If $X$ is planar, then $X$ is an AR-space iff $X$ is an AARspace.

Proof of Corollary 1. Let $X$ be a 1-dimensional AAR-space. By Theorem $2, X$ is a dendrite. Since the 1-dimensional AR-spaces coincide with the dendrites [1, p. 138], Corollary 1 follows.

Proof of Corollary 2. Let $X$ be a subset of the plane $E^{2}$ which is an AAR-space. By Theorem $2, X$ is a locally connected continuum which does not separate $E^{2}$. Therefore $X$ is an AR-space [1, p. 132], and Corollary 2 follows.

Remark. In [6] C. W. Saalfrank generalizes the notion of an absolute retract for compact Hausdorff spaces to a concept which he calls an absolute homotopy retract (abbreviated AHR). It is easy to see that every contractible continuum is an AHR, and conversely, by taking the cone over an AHR, one sees that every AHR is contractible. Thus for the case of compact metric spaces, we see that the class of AAR-spaces is a proper subclass of the class of AHR-spaces.

\section{REFERENCES}

1. K. Borsuk, Theory of retracts, Monografie Mat., tom 44, PWN, Warsaw, 1967. MR 35 \#7306.

2. O. Hanner, Retraction and extension of mappings of metric and non-metric spaces, Ark. Mat. 2 (1952), 315-360. MR 14, 396; 1278. 
3. S. T. Hu, Homotopy theory, Pure and Appl. Math., vol. 8, Academic Press, New York and London, 1959. MR 21 \#5186.

4. S. Kinoshita, On some contractible continua without fixed point property, Fund. Math. 40 (1953), 96-98. MR 15, 642.

5. J. M. Kysko, An example of a contractible continuum without fixed point property for homeomorphisms, Bull. Acad. Polon. Sci. Sér. Sci. Math. Astronom. Phys. 20 (1972), 663-666. MR 47 \#7720.

6. C.W. Saalfrank, A generalization of the concept of absolute retract, Proc. Amer. Math. Soc. 12 (1961), 374-378. MR 23 \#A2858.

7. S. Willard, General topology, Addison-Wesley, Reading, Mass., 1970. MR 41 \#9173.

DEPARTMENT OF MATHEMATICS, UNIVERSITY OF SASKATCHEWAN, SASKATOON, CANADA S7N OWO 